\title{
The Vulcanoid Asteroids: Past, Present and Future
}

\author{
Martin Beech ${ }^{1,2}$, Lowell Peltier ${ }^{2}$ \\ ${ }^{1}$ Campion College, The University of Regina, Regina, Canada \\ ${ }^{2}$ Department of Physics, The University of Regina, Regina, Canada
}

Email address:

beechm@uregina.ca (M. Beech), Peltier21@uregina.ca (L. Peltier)

\section{To cite this article:}

Martin Beech, Lowell Peltier. The Vulcanoid Asteroids: Past, Present and Future. American Journal of Astronomy and Astrophysics. Vol. 5, No. 3, 2017, pp. 28-41. doi: 10.11648/j.ajaa.20170503.12

Received: July 10, 2017; Accepted: July 25, 2017; Published: August 25, 2017

\begin{abstract}
A review and discussion of both the historical and contemporaneous ideas pertaining to the putative population of Vulcanoid asteroids is presented. Current observations indicate that no objects larger than between 5 to $10 \mathrm{~km}$ in diameter reside in the orbital stability zone between 0.06 and $0.2 \mathrm{AU}$ from the Sun, and that, at best, only a small population of Vulcanoid asteroids might exist at the present epoch. We review the physical processes (sublimation mass loss, evolution of the Sun's luminosity, Poynting-Robertson drag, the Yarkovsky effect, the YORP effect, unipolar heating and collisions) that will control the lifetime against destruction of objects, either primordial or present-day, that chance to reside in the Vulcanoid region. It is argued that there are no overriding and/or absolute physical mechanisms that fully rule-out the present-day existence of a small Vulcanoid population, but we note that the gap between what the observations allow and what the theoretical models deem possible is closing rapidly.
\end{abstract}

Keywords: Vulcanoid Asteroids, Orbital Evolution, Thermal Processing, Detection Methods

\section{A brief Historical Introduction}

That there was a problem in accounting for the observed perihelion advancement of Mercury in terms of the gravitational perturbations afforded by the known planets was first articulated by Urbain Le Verrier in 1859 [1]. Of the (then) deduced 565 arc seconds per century advancement in Mercury's perihelion, all but 38 arc seconds per century could be accounted for - mostly in terms of perturbations due to Venus, Earth and Jupiter. The anomalous 38 arc seconds per century (reckoned to be 43 arc seconds per century in the modern era) brought into question the then prevalent Laplacian ideal of dynamical determinism, and it even prompted questions concerning the universal applicability of Newton's inverse square law of gravitational attraction [2, 3, 4]. Le Verrier's response to the Hermian perihelion advancement anomaly, however, was to invoke, in similar vein to his earlier solution to the anomalous motion of Uranus [3, 5], a new planet, or numerous "corpuscles" (that is, a ring of asteroids), interior to the orbit of Mercury. This body (or collection of bodies) would accordingly be constrained, in mass and heliocentric distance, so as to provide the additionally required
Hermitian gravitational perturbation [1]. Le Verrier argued that if the additional perturbing object were a single planet, with an assumed circular orbit of radius $0.14 \mathrm{AU}$, it must have an associated mass of order $1 / 17^{\text {th }}$ that of Mercury. Such an object, having the same bulk density as Mercury, would be nearly 1900 kilometres across. This result, of course, was problematic since such a massive (and accordingly large) body, although on an orbit close-in towards the Sun, should none-the-less be readily observable. Confident in the absolute applicability of Newton's inverse square law, however, and the earlier success in predicting the existence of Neptune through the observed orbital residuals in the position of Uranus [5], astronomers readily accepted Le Verrier's conjecture and even christened the postulated new planet with the name Vulcan - after the Roman god of fire, metalwork and the forge, and the erstwhile husband of Venus.

With the idea of a new planet, or swarm of asteroids, existing interior to the orbit of Mercury seemingly established, professional and amateur astronomers alike soon set-out to integrate the new object(s) within the long- 
established framework of the Titius-Bode law [6]. Indeed, while it is still not known why the Titius-Bode law is so successful, it was certainly the case that its efficacy had been well established by the mid $-19^{\text {th }}$ century. This came about through its successful accommodation of the discovery of Uranus by William Herschel in 1782, the identification of main-belt asteroids in the gap between Mars and Jupiter, with Ceres being discovered by Giuseppe Piazzi in 1801, and, perhaps less successfully, it accommodated the discovery of Neptune by Johann Galle in 1846 - the latter discovery being predicated on the successful (although largely fortuitous) mathematical predictions by Le Verrier and John Couch Adams. The original Titius-Bode law [6, 7] constructed by Johann Titius in 1766 was based upon the number sequence $0,3,6,12$, $24,48 \ldots$ and so on, with each number in the sequence (except the second one) being twice that of its forerunner. To each of these sequence numbers Titius then added 4 and divided the sum by 10 to 'determine' the orbital radius of each planet in astronomical units: $0.4,0.7,1.0,1.6,2.8$, $5.2, \ldots$ and so-on. It is the apparent simplicity of the TitiusBode law that underlies its utility, but it affords no obvious explanation for an intra-mercurial planet. This being said, in 1878 , B. G. Jenkine [8] found a numerical 'fix' to make the historic sequence 'work'. Jenkine argued that the primary number sequence given by Titius should really start at -3 rather than 0 , and accordingly by adding 4 and dividing by 10 , one acquires a new first orbit at 0.1 astronomical units this implies a deviation error of 29 percent with respect to Le Verrier's adopted value $0.14 \mathrm{AU}$; a value no more at odds with the predicted and actual semi-major axis of Neptune's orbit, which also comes-in with a $29 \%$ deviation. Jenkine writes, "may not the number 1 [term giving a radius of $0.1 \mathrm{AU}]$ represent the distance to Vulcan, or more probably the mean-distance of a ring of asteroids of which Vulcan is the brightest?" [8]. Interestingly, Jenkine finished his letter with some speculations on the structure of the outer regions of the solar system. Starting with Ceres, in the main-belt asteroid region, Jenkine noted that moving inwards towards the Sun, there are three planets and then the Vulcanoid asteroids. Moving outwards from Ceres, Jenkins, additionally noted, that there are another three planets, accordingly, by invoking a symmetry argument, he reasoned that beyond Neptune there must be, "a ring of asteroids at an average distance of 772 [that is, at a distance of 77.2 astronomical units]. Jenkine then continues, "Perhaps the optical instruments of the future may help to answer this question: is there a ring of asteroids beyond Neptune?" [8]. Incredibly, then, Jenkine not only adapts the Titius-Bode law to accommodate the existence of a Vulcanoid asteroid belt, but he also anticipates, by more than one-hundred years, the discovery of the Kuiper belt. Equally remarkable is the point that Jenkine's estimate of $0.1 \mathrm{AU}$ for the location of the Vulcanoid asteroids is entirely consistent with present-day computer simulations (see below), in that a long-lived orbital stability zone does exist in the region between 0.09 and 0.20 AU from the Sun.
As with all applications of the Titius-Bode law we cannot take any of its apparent predictions, and/or successes, seriously. The law, or more correctly rule, has no physical foundation [6] and without any underlying theory for the origin of the underlying number sequence it is nothing but a façade - a convenient piece of numerology with no actual foundation in physical fact. Indeed, this very point was explored by philosopher Georg Hegel in his 1801 doctoral thesis, Dissertatio philosophica de orbitsis planetarum, where he contrasted the Titius-Bode law against the socalled lambda-sequence of numbers (which is an interweaving of the sequences $2^{n}$ and $\left.3^{n}, n=0,1,2, \ldots\right)$ described circa 360 B. C. by Plato in his classic work Timaeus. The point being, as Hegel reasoned, one can posit almost any sequence of numbers to describe the observations relating to planetary spacing and argue for specific hits and misses according to special conditions and one's specific philosophical bent. Indeed, one can always invoke something akin to Wittgenstein's finite rule paradox to save the day. This latter approach was actually applied by American astronomer Pliny Earle Chase in an intriguing article written in 1873 [9]. Using rather obscure (if not entirely incorrect) physical arguments, Chase developed a planetary spacing rule according to the representation: $R_{n}=$ $(\pi / 32)(1+n \pi)$, where $n=1,2,3,5,9,17,33,65,97$. The orbit for Mercury follows from $n=1$, giving $R_{1}=0.407$, and $n$ increases according to the rule $n_{\mathrm{i}}=\left(2 \mathrm{n}_{\mathrm{i}-1}-1\right), i=1$, $2,3, \ldots, 8$ until the $9^{\text {th }}$ planet, where, rather than taking $n_{9}=$ 129 according to the number increase rule, it is arbitrarily set equal to 97 in order to afford a good fit to the observed orbital semi-major axis of Neptune: $R_{9}=30.015$ (the TitiusBode law for Neptune gives $R_{9}=38.8$ ). Just as the TitiusBode law was able to accommodate the discovery of Uranus and the asteroid belt, so Chase's rule can accommodate the discovery of Pluto by setting $n=127$, again breaking away from the step-by-step rule for the evaluation of $n$. For Chase it was enough that his formula for $R_{n}$ could be made to work, but at the sacrifice that after the $n=8$ planet (Uranus) it is no longer predictive. Interestingly, Chase's rule can accommodate the presence of Vulcan by simply setting $n=0$, and obtaining $R_{0}=0.098$, once again, however, this treatment ignores the ordered number sequence which describes the orbital radii of Mercury through to Uranus.

The inherent philosophical impotency of the Titius-Bode law was numerically demonstrated in a thoughtful paper by Hayes and Tremaine [10] published in 1998. These authors found from the study of randomly generated orbital distributions that the most important factor in determining system stability was that planets be regularly spaced at distances which exceed the maximum sum of any two adjacent planet's Hill radii. Moving beyond the simple numerology of the Titius-Bode rule, Schumacher and Gay [11] where meaningfully prompted, according to predictions based upon scale relativity theory, to search for Vulcanoid asteroids within the data archive of the Solar and Heliospheric Observatory (SOHO) spacecraft. Scale 
relativity theory, as first introduced by Laurent Nottale (CNRS, Meudon), was developed as a means of melding classical relativity theory to a fractal space-time framework, and it is offered as a generalization of quantum mechanics to the macroscopic world. Indeed, the theory predicts that gravity will tend to quantize the distribution of objects within the universe, and it specifically predicts for the solar system a planet spacing rule of $a \approx 0.04(n+1 / 4)^{2}$, where $a$ is the semi-major axis in astronomical units and $n=1,2,3, \ldots$ etc. Schumacher and Gay [10] specifically note that the $n=$ 1 and $n=2$ positions within the solar system are not filled (the orbit of Mercury corresponds to the $n=3$ term), and that these two locations fall at distances of 0.06 and 0.20 AU from the Sun - a region exactly coincident, it turns out, with the putative orbital stability zone for the Vulcanoids. The SOHO data search by Schumacher and Gay revealed no Vulcanoid objects brighter than visual magnitude of +7 , corresponding to an upper size limit of about $60 \mathrm{~km}$. At present scale relativity remains highly conjectural, and it is certainly not a proven or even well-received theory, but in contrast to the Titius-Bode law it makes predictions based upon an underlying physical argument.

In spite of numerous searches and several false detections $[4,12,13]$, it was essentially apparent from the 29 July 1878 solar eclipse onwards that no large planet interior to the orbit of Mercury existed. Indeed, by 1926, Russell, Dugan and Stewart in their widely read text Astronomy [14], were to conclude with a degree of authority, based upon photographic survey plates obtained during the Lick Observatory eclipse expeditions of 1901, 1905 and 1908, that, "there are no intra-Mercurial bodies brighter than the eighth magnitude, that is, more that about 30 miles [48-km] in diameter". Charles Young in his A Text-book of General Astronomy [15], first published in 1899, (the book that Russell, Dugan and Stewart, in fact, set out to revise) commented that, "it is extremely probable that there are a number, and perhaps a very great number, of intraMercurial asteroids". Indeed, from the turn of the $19^{\text {th }}$ century onwards, the astronomical literature [see $4 \& 12$ for extensive survey references] indicates that while it was accepted that Vulcan the planet was a chimera, the existence of Vulcanoid asteroids was all but guaranteed.

The very reasons for invoking the existence of an intraMercurial object (either as a planet or an extensive body of asteroids) were ultimately rendered obsolete by Albert Einstein. Through the publication of his founding papers on general relativity, starting in 1915 , it was shown that the anomalous 43 arc seconds per century advancement of Mercury's perihelion was explainable as a general relativistic correction to Newtonian theory [4]. In spite of Einstein's work forging a fundamentally new explanation for Mercury's observed advancement of perihelion, the notion that a population of intra-Mercurial asteroids might still exist, although no longer required to produce any dynamical perturbation effects, has persisted. Indeed, the search for objects orbiting close-in towards the Sun has continued right-up to the modern era $[4,16,17,18,19]$, although no definitive Vulcanoid object has, as yet, been identified. While Russell, Dugan and Steward placed an upper size limit of $48 \mathrm{~km}$ to the diameter on any potential Vulcanoid asteroid in 1926, more recent surveys have reduced this upper size limit by nearly a factor 10; Steffle et $a l$., [20] concluding, for example, that, "there are presently no Vulcanoids larger than $5.7 \mathrm{~km}$ in diameter".

In spite of continued observational efforts, the existence, or not, of Vulcanoid asteroids has remained stubbornly unresolved. At best, it would appear, there may be a small population of objects located within the Vulcanoid orbital stability zone at the present epoch. Equally, it is entirely possible that absolutely no Vulcanoid asteroids exist at the present time. It is additionally unclear if even a primordial population of Vulcanoid objects ever existed. The latter situation, in principle, might be investigated via the detailed analysis of the Hermian crater size-frequency-distribution (SFD), but even with the greatly improved dataset provided for by the recent MESSENGER spacecraft, there is no clear signature of any prolonged or additional cratering over and above that which might be expected from the cratering records observed for the Moon and Mars [21]. The most heavily cratered Hermian domains indicate a cratering SFD consistent with that expected from the epoch including the Late Heavy Bombardment (LHB), but the subsequent volcanically-driven emplacement of extensive inter-crater plains has greatly confused the overall situation. Accordingly, it is not readily clear, from any observational dataset, that there must have been an extensive population of Vulcanoid asteroids in the distant past.

Amongst the many exoplanetary systems that have now been identified, a significant number of tightly-packed planetary (TPP) systems, containing 3,5 or even 7 terrestrial planets, have been identified, and characteristically these systems span a region of space that is no larger than the orbit of Mercury about our Sun [22]. It has been suggested that such TPP systems are ultimately unstable, and that mutual gravitational interactions will result in the ejection of some planets and the collisional destruction of others. Accordingly, it may be the case that many planetary systems, including our own, formed in a TPP configuration and only later evolved into a system containing fewer close-in planets. Importantly for our narrative, during the later stages of TPP system evolution, it is likely that a population of asteroid-like bodies, situated close to the parent star, will develop. From the foregoing, therefore, it may be argued that there are at least two good reasons why the solar system might have supported a primordial population of Vulcanoid asteroids - either as the result of direct planetesimal growth, or via the destruction of two or more closely-packed planets. To these scenarios we can additionally add the process of Hermian Trojan capture [23], as well as the inward evolution of cometary nuclei, near-Earth asteroids and assorted collisional ejecta from the outer solar system [24].

Evans and Tabachnik [25] first showed in 1999 that there was an orbital stability zone interior to that of planet 
Mercury. Indeed, numerical simulations revealed the existence of an annulus between 0.1 and $0.19 \mathrm{AU}$, which Evans and Tabachnik described as, "one of the most dynamically stable regions in the entire Solar System". The outer limit of the orbital stability zone is set according to the attainment of a Mercury crossing orbit on a timescale short compared to the age of the solar system, while the inner edge simply corresponded to the smallest orbit considered in the calculations - in principle orbits as small as $a \sim 0.01 \mathrm{AU}$, corresponding to the Sun's Roche limit, might be considered (although see later). A second more detailed study by Evans and Tabachnik [26], in 2002, confirmed their preliminary analysis (slightly expanding the size of the stability zone, however, to between 0.09 and $0.20 \mathrm{AU}$ ) and this provides us with some hope that a population of Vulcanoid asteroids, primordial or otherwise, might exist at the present epoch. This hope draws primarily upon the observation that in every other dynamically stable zone within the solar system there is a population of associated objects. This being said, the results presented by Evans and Tabachnik [25, 26] are based purely upon standard Newtonian N-body dynamics, and no nongravitational perturbation effects, such as sublimation mass loss, collisions and/or radiative interactions were included in their analysis - such considerations will be presented in sections 3 and 4 below.

That the Vulcanoid zone is far from being a dynamically inactive region of the solar system is evidenced by the fact that both asteroids and cometary nuclei are regularly observed to pass through its borders in the modern era [23]. A search of the JPL Small-Body database, for example, reveals that at the present epoch there are 244 asteroids that cross the orbit of Mercury with perihelion distances located within the Vulcanoid zone. While the capture of a cometary nucleus, or an asteroid, into the Vulcanoid zone is highly unlikely, it is not altogether impossible. Likewise material ejected from the surface of Mercury, as a result of surface impacts, may potentially acquire a stable orbit within the Vulcanoid region. Indeed, substantial impacts have been invoked by Wieczorek et al., [27] as an explanation for the un-anticipated 3:2 spin-orbit resonance of Mercury, and such impacts, of course, will launch substantial amounts of debris into the near-Hermian environment. Additionally, the massive Caloris impact basin, with a diameter of some $1,450 \mathrm{~km}$, is estimated to have formed some 3.7 billion years ago and accordingly some of the material from that impact could have found a stable haven within the Vulcanoid zone.

The Vulcanoid zone, being set so close to the Sun, makes it an extremely difficult region to study both from the ground and from space. The historical surveys from the ground have been conducted predominantly at optical wavelengths, and at times of total solar eclipse. Leake et al [17], in contrast, conducted between 1979 and 1981 a ground-based survey for Vulcanoids at infrared wavelengths. Usefully, as Leake et al. [17] pointed-out, at L band wavelengths $(\lambda \sim 3.5 \mu \mathrm{m})$ survey work can be conducted during daylight hours and more importantly, the L-band is sensitive to the peak emission expected for a blackbody radiator of temperature $850 \mathrm{~K}$ - the equilibrium temperature expected of objects located at about $0.1 \mathrm{AU}$ from the Sun (see below). Working to a limiting magnitude of +5 , Leake et al. found no Vulcanoid candidates in their survey region, and concluded that no Vulcanoids larger than about $50 \mathrm{~km}$ in diameter exist at the present epoch. The surveys conducted from space, using SOHO and STEREO archive data $[10,18,20]$, have all been made with instruments not optimized for the detection of small, rapidly moving bodies orbiting close to the Sun. Next generation survey satellites, however, such as the proposed infraredwavelength Sentinel mission [28], designed specifically to search for near-Earth asteroids, may well be better suited to detecting Vulcanoids. Likewise the Solar Orbiter, recently selected as part of ESA's Cosmic Vision Program, and due for launch in 2018, will be dedicated to the study of solar and heliospheric phenomena and may additionally be well positioned to search for kilometre-sized Vulcanoid objects. Likewise, we are also on the threshold of an era in which space flights, such as those proposed by Virgin Galactic, will begin to become increasingly affordable and common place, and it may be possible (with appropriate instrumentation) to use such regular excursions into low Earth-orbit to automatically search for objects orbiting close to the Sun.

\section{The Post-20 ${ }^{\text {th }}$ Century Situation}

With the announcement of funding for the Mercury Surface, Space Environment, Geochemistry and Ranging (MESSENGER) mission in 1999, some considerable interest was once again directed towards the Vulcanoid asteroids. In particular two important papers by Stern and Durda [29] and Vokrouhlicky, Farinella and Bottke [30] were published in 2000. Stern and Durda examined various scenarios for the collisional evolution of objects located within the Vulcanoid zone, concluding that due to high encounter speeds survival on timescales comparable to the age of the solar system was highly unlikely (see, however, section 6 below). Indeed, Stern and Durda argued that, "it is plausible that the entire region is virtually empty of kilometre-scale and larger objects". Vokrouhlicky, Farinella and Bottke, in contrast, considered the potential depletion of smaller objects from within the Vulcanoid zone through the action of radiative drag forces. Finding such mechanisms to be highly efficient, the latter authors argued that objects smaller than a kilometre or so in diameter would be rapidly lost from the Vulcanoid region, and concluded, "Collisions probably eliminated most larger $(D>1 \mathrm{~km})$ vulcanoids, while Yarkovsky drift removed the smaller objects $(D<1$ $\mathrm{km})$. Thus, the sole remaining evidence supporting the existence of the vulcanoid populations at any time is contained in Mercury's enigmatic crater record". Post-2000 the consensus has been mostly against the existence of any substantive population of Vulcanoids, not only at the 
present but at any time in the past. Much has changed during the past 15 years, however, with respect to our understanding of asteroid structure and with respect to the effects of non-gravitational phenomena on asteroid dynamics, and it is with such thoughts in mind that this present study has been initiated. In the sections that follow it is our intention to present a systematic study of those factors that can affect the survivability lifetime of objects located within the hypothetical Vulcanoid region, situated between 0.06 and $0.2 \mathrm{AU}$ from the Sun, and to see how past analysis stands up to present-day scrutiny.

\section{Sublimation and the Sun's Variable Luminosity}

In an earlier paper the authors [24] have argued that the inner edge of the Vulcanoid zone can be set at a heliocentric distance of $0.06 \mathrm{AU}$. While this distance was taken as an extreme value that encompassed previously published estimates within the literature, it is ultimately a distance determined by sublimation mass loss. The authors have investigated the effects of mass loss via sublimation in the case of then putative, but now rejected, terrestrial planet in orbit about the star $\alpha$ Centauri B [31], and the same equations and analysis will be applied here to the Vulcanoids. The relevant equations for the equilibrium temperature and change in physical radius are

$$
T=278\left(\frac{(1-A) L(t)}{\varepsilon D^{2}}\right)^{1 / 4}
$$

and

$$
\frac{d R}{d t}=-\frac{\sigma(T)}{\rho_{B}}
$$

where $T, A, D$ and $\varepsilon$ are the temperature, albedo, orbital radius and emmissivity of the Vulcanoid, and $L(t)$ is the time dependent luminosity of the Sun. The time variation in the asteroid radius $R$ is related to the surface mass loss rate $\sigma(T)$ and the composition-dependent bulk density $\rho_{B}$. The albedo and emmissivity will be assumed constant in this analysis, and we adopt $A=0.3$ and $\varepsilon=0.9$ as characteristic values. The luminosity and orbital radius in equation (1) are to be expressed in solar units and astronomical units respectively. The surface mass loss rate $\sigma(T)$ is determined through Langmuir's equation and a suitable, composition-specific, phase-equilibrium vapor pressure formulation. In the discussion below we shall consider two representative compositions for the Vulcanoids: iron $(\mathrm{Fe})$, and fayalite $\left(\mathrm{Fe}_{2} \mathrm{SiO}_{4}\right)$ - see table 2.1 of reference [31] and the sections below for specific material properties. The variation with age of the Sun's luminosity (figure 1) is based upon a $4^{\text {th }}$-order polynomial least-square fit to a solar mass model obtained from the EZ-web [32].

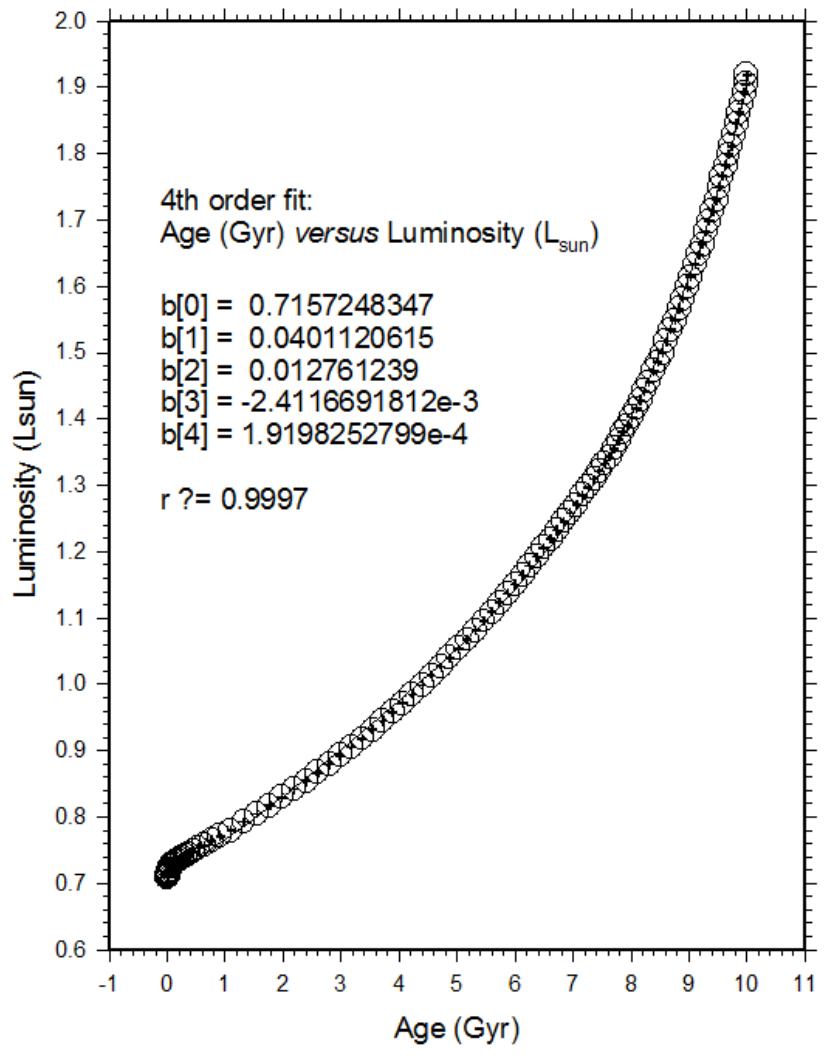

Figure 1. Luminosity (in solar units) versus age (in Gyr) for a 1 solar mass stellar model. The coefficients to the $4^{\text {th }}$-order polynomial least-squares fit to the model data points are shown to the upper left of the diagram. Model data from the EZ-web server [32].

Figure 1 reveals that the Sun's luminosity has changed by some 25 to 30 percent over the age of the solar system, and this, as will be seen, significantly changes the lifetime against sublimation of any putative Vulcanoids, and it also indicates that the inner edge of the Vulcanoid zone will have moved outwards over time. Figure 2 shows a comparison of lifetime against sublimation mass loss for Vulcanoids with initial sizes between 1 and $150 \mathrm{~km}$ at a heliocentric distance of $0.06 \mathrm{AU}$ (the adopted inner edge of the Vulcanoid zone at the present epoch). The consequences of assuming a constant solar luminosity are now apparent, and figure 2 reveals that when a more realistic variation in solar luminosity is allowed for the survival time against sublimation mass loss is significantly increased for iron Vulcanoids of all sizes, and for stony (fayalite composition) Vulcanoids with initial diameters smaller than about $80 \mathrm{~km}$. For stony (fayalite) Vulcanoids initially larger than $80 \mathrm{~km}$ in diameter, the lifetime against mass loss by sublimation is reduced under the time variable luminosity scenario, since for these objects $L(t)>1 \mathrm{~L}_{\odot}$ once $t>4.5 \mathrm{Gyr}$. At $10 \mathrm{~km}$ initial diameter the survival lifetime against mass loss by sublimation is increased by 1.45 billion years for iron Vulcanoids, and 3.28 billion years for stony (fayalite) Vulcanoids. While no iron Vulcanoids survive to the present age of the solar system under the $L(t)=1 \mathrm{~L}_{\odot}$ scenario, those initially larger than $140 \mathrm{~km}$ in diameter can survive under the more realistic variable $L(t)$ model. Indeed, under the variable $L(t)$ model, an iron Vulcanoid initial $150 \mathrm{~km}$ across 
will have a diameter, at the present epoch $(t=4.56 \mathrm{Gyr})$, of about $3 \mathrm{~km}$. Similarly, stony (fayalite) Vulcanoids initially larger than about $15 \mathrm{~km}$ in diameter could survive against sublimation mass loss for $t>4.56$ Gy under the variable $L(t)$ scenario.

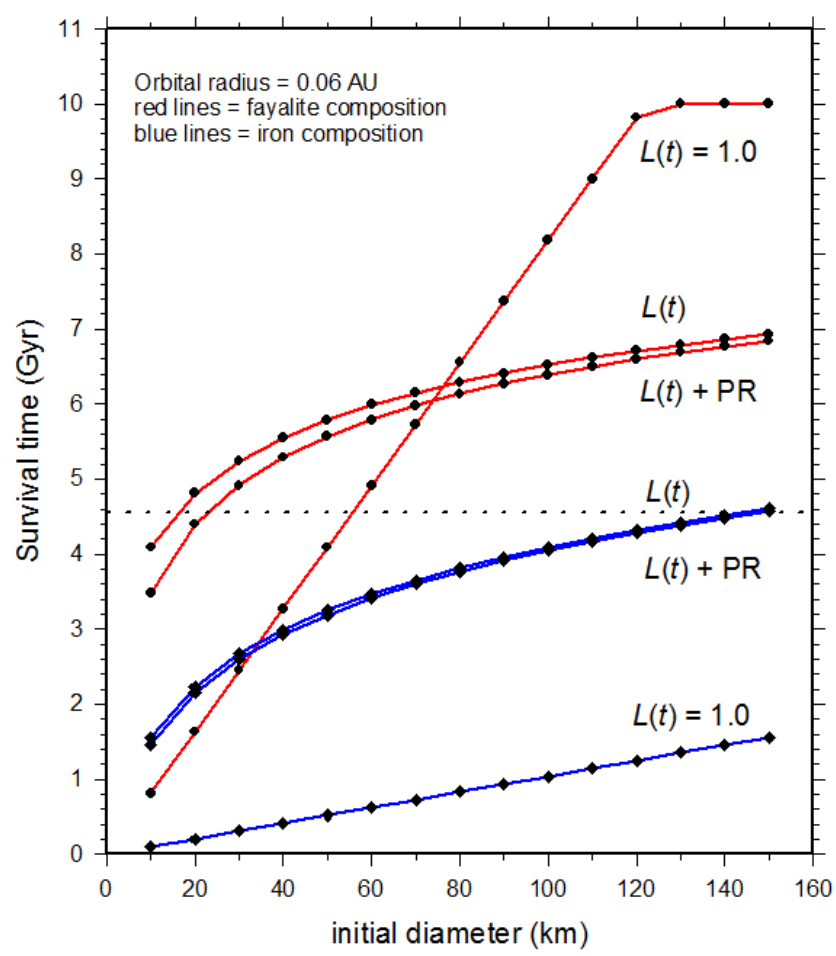

Figure 2. Lifetime against sublimation mass loss for Vulcanoids with initial sizes from 1 to $150 \mathrm{~km}$ and an initial orbital radius of $0.06 \mathrm{AU}$. Red lines are for a stony (fayalite) composition, while blue lines correspond to a pure iron composition. The curves are labelled according to the solar luminosity law that has been applied. The curves labelled with $+P R$ allow for orbital evolution via Poynting-Robertson drag (see section 4). The horizontal dotted line indicates the 4.56 billion year age of the solar system.

\section{Interactions with the Solar Radiation Field}

The two main effects that can modify the orbit of small objects in motion about the Sun are Poynting-Robertson drag [33] and the Yarkovsky effect [34, 35]. The former effect results in the gradual inward motion of an object as it absorbs energy and momentum from the Sun's radiation field. The latter effect is due to a recoil action resulting from the anisotropic reemission of thermal radiation by a spinning body, and according to the physical circumstances (primarily variations in the obliquity of the object's rotation axes) this effect may result in either an inward or outward motion.

The Poynting-Robertson drag acts to reduce a body's orbital angular momentum, and enters into the equation of motion via a reduction in the gravitational constant term $\mu$ such that $\mu=G M_{\odot}-\alpha c$, where $G$ is the universal gravitational constant, $M_{\odot}$ is the Sun's mass, $c$ is the speed of light, and $\alpha$ accounts for the outwardly directed radiation pressure. Wyatt and Whipple [33] show that $\alpha=3 L_{\odot} /(16 \pi$
$R c^{2} \rho$ ) where $L_{\odot}$ is the Sun's luminosity, and where $R$ and $\rho$ are the body's radius and density. The Poynting-Robertson drag acts to circularize an object's orbit by forcing the orbital eccentricity towards zero, and it additionally acts to reduce the orbital semi-major axis $a$. For nearly circular orbits, the time for orbital decay, $T_{P R}$, is given by the relation

$$
T_{P R}=\frac{a^{2}}{4 \alpha} \approx 7 \times 10^{5} R \rho a^{2}
$$

where in the second term on the rhs of (3) the semi-major axis is expressed in astronomical units and the timescale is in years - additionally, the radius is expressed in metres and the density is in kilograms per metre cubed. Setting the Poynting-Robertson decay time to the age of the solar system, $T_{P R}=4.5 \times 10^{9}$ years, a condition upon the initial size, composition and initial orbit results in the inequality $R \rho>$ $8428.6 / a^{2}$ (AU). The density term will vary from of order $4000 \mathrm{~kg} / \mathrm{m}^{3}$ for fayalite to of order $8000 \mathrm{~kg} / \mathrm{m}^{3}$ for iron, and characteristically, therefore, to survive against orbital decay due to Poynting-Robertson drag, over the age of the solar system, a Vulcanoid must have an initial diameter $d(\mathrm{~m}) \approx 2.1$ $/ a^{2}$ (AU). For an initial orbital radius of $0.06 \mathrm{AU}$, therefore, to survive against Poynting-Robertson drag over the age of the solar system (assuming at this stage no sublimation mass loss and a constant luminosity for the Sun), a Vulcanoid asteroid will need to have an initial diameter greater than $583 \mathrm{~m}$ - for initial orbital radii of $0.15 \mathrm{AU}$ and $0.25 \mathrm{AU}$ the initial diameter will need to exceed $93 \mathrm{~m}$ and $34 \mathrm{~m}$ respectively.

For circular orbits, the rate of change of the orbital radius due to Poynting-Robertson drag is [33]

$$
\frac{d a}{d t}=-\frac{2 \alpha}{a}
$$

and this equation can be simultaneously solved for with equations (1) and (2) to follow the change in orbital radius resulting from Poynting-Robertson drag including the effects of sublimation and the time variation of the Sun's luminosity - note that in this case the $\alpha$ term is additionally time variable due to its dependency upon the solar luminosity and the radius of the Vulcanoid body. Figure 3 shows the survival times for Vulcanoids in the size range of 100 meters to $5 \mathrm{~km}$. As we would expect, the survival time of iron Vulcanoids will always be less than that of a same sized, same initial orbit Vulcanoid composed of fayalite. Likewise, the shortest survival times are exhibited by the smallest Vulcanoids [as to be expected from equation (3)]. For a primordial iron Vulcanoid to survive to the present time it will need to have an initial radius larger than $0.5 \mathrm{~km}$ and be located further than 0.1 AU from the Sun. At 0.15 AU initial orbit radius, all iron Vulcanoids larger than 100 meters in diameter will survive to the present epoch. 


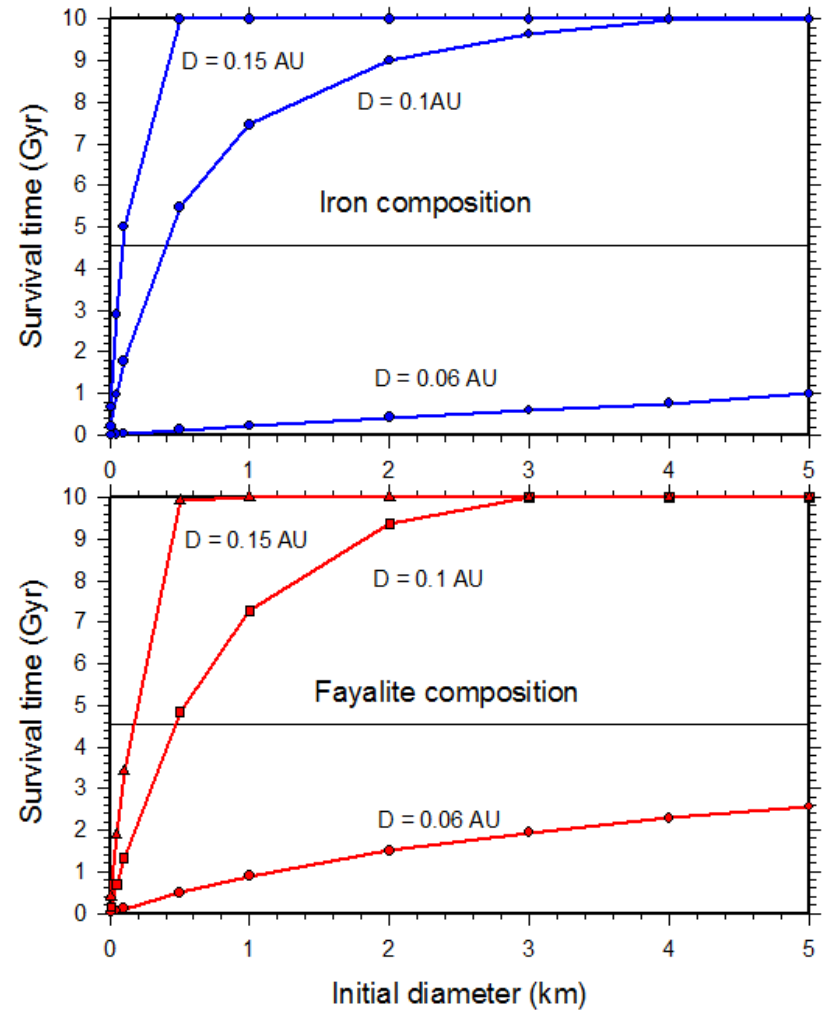

Figure 3. Survival times for primordial Vulcanoids including the variation in the Sun's luminosity with age, sublimation mass loss and PoyntingRobertson drag. Top: iron composition objects. Bottom: fayalite composition objects. The thin horizontal line indicates the present age of the solar system.

Anticipating the discussion to be presented in section 6, where the effects of collisions are to be addressed, figure 4 shows that the survival times for fayalite composition Vulcanoids, with varying initial sizes, initial orbit location and starting (or injection) epoch. Three starting times are considered, with a primordial population starting at $t$ (start) $=$ 0.0 , an intermediate generation of objects starting at $t$ (start) $=2.0 \mathrm{Gyr}$, and a current epoch generation of objects starting at $t$ (start) $=4.5$ Gyr. The primary reason why the survival time decreases, for a given initial size and orbital radius, is the increase in the Sun's luminosity with age - the increasing luminosity factor drives an enhanced sublimation mass loss rate per unit time interval [as seen from equations (1) and (2)], the increased luminosity and the small radius additionally work to increase the $\alpha$-term in the PoyntingRobertson drag equation, and this, as indicated by equation (4), acts to reduce the orbital radius.

In the size range considered in figures 3 and 4, 100 meters to $5 \mathrm{~km}$, the clearing time due to Poynting-Robertson drag is extremely short for an initial orbital radius of $0.06 \mathrm{AU}$, with all survival times being shorter than half the present age of the solar system. For initial orbital radii of 0.1 and $0.15 \mathrm{AU}$, a primordial fayalite Vulcanoid larger than $100-\mathrm{m}$ in diameter could in principle survive to the present epoch. For a starting time epoch corresponding to $t=2.0 \mathrm{Gyr}$, a fayalite Vulcanoid larger than 200 meters diameter could survive against Poynting-Robertson drag and sublimation mass loss into the present era. Vulcanoids larger than $600 \mathrm{~m}$, situated at 0.15
AU at the present epoch, can survive against destruction for the remainder of the Sun's main sequence lifetime.

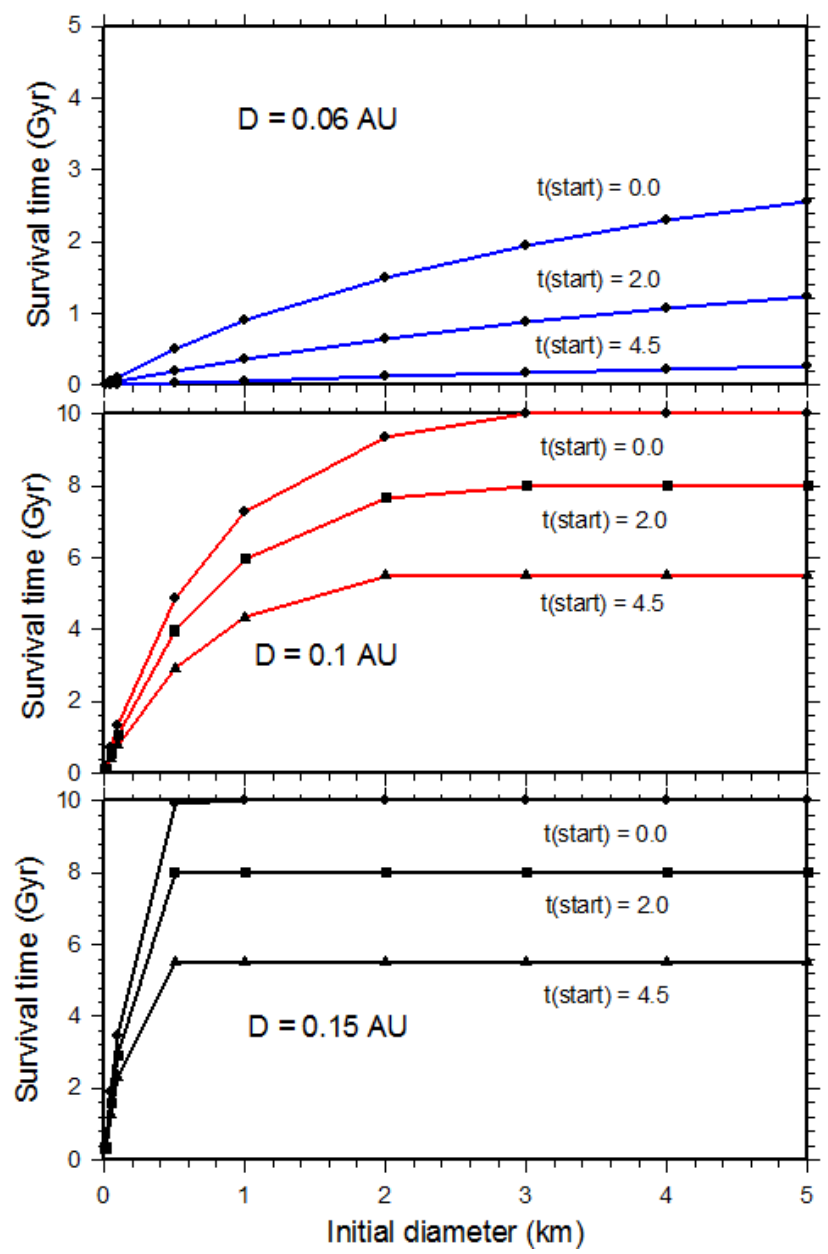

Figure 4. Survival times according to initial size, orbital distance, and starting epoch. A fayalite composition is assumed, and three starting times are considered corresponding to a primordial population with $t$ (start) $=0.0$, an intermediate generation of objects produced at $t$ (start) $=2.0 \mathrm{Gyr}$, and a current epoch generation with $t$ (start) $=4.5$ Gyr. The calculations in each case are stopped once the Sun achieves an age of $10 \mathrm{Gyr}$, at which time it is deemed that our L $(t)$ approximation no longer holds, and at which point the main-sequence lifetime of the Sun is over. Indeed, once the Sun begins to evolve towards a red giant configuration the entire inner-solar system region to near 1 AU will be subsumed into its outer layers.

While the effects of Poynting-Robertson drag are more keenly felt by smaller sized objects located close-in towards the Sun, as indicated by the radius $R$ term in equation (3), the orbits of more substantive objects at large heliocentric distances are affected by Yarkovsky drift. The equations describing the secular change in the orbital semi-major axis due to Yarkovsky drift have been previously described in great detail $[34,35]$, but of specific concern here is the time evolution of the orbital semi-major axis, which is written as:

$$
\frac{d a}{d t}=-\frac{4}{9} \frac{(1-A) \Phi}{n}\left[W\left(R_{n}, \Theta_{n}\right) \sin ^{2} \gamma-2 W\left(R_{\omega}, \Theta_{w}\right) \cos \gamma\right]
$$

where $A$ is the albedo, $n$ is the orbital mean motion, $\gamma$ is the obliquity of the spin axis, $\omega$ is the rotational frequency, and 
where

$$
\Phi=\pi R^{2} \frac{F}{m c}
$$

with $R$ and $m$ being the radius and mass of the body, $c$ is the speed of light and $F=L_{\odot} / 4 \pi D^{2}$ is the solar radiation flux at orbital distance $D$. The two $W$-functions on the rhs of (5) account for the seasonal and the diurnal components of the Yarkovsky effect respectively, and they are in turn determined by the thermal parameters of the body along with an assumption about the rotational frequency $v$, for the diurnal component, and the enumeration of the orbital mean motion $n$ in the case of the seasonal component - see [36] for a full discussion of the $W$-functions. The thermal properties of the object enter into equation (5) via the non-dimensional radius terms $R_{n}$ and $R_{\omega}$, where parametrically, $R_{v}=R / l_{v}$, and

$$
l_{v}=\sqrt{K /(\rho C v)}
$$

with $K$ being the surface thermal conductivity, $C$ the specific heat capacity and $\rho$ the density of the body. For an iron composition, $l_{v}$ is of order $25 \mathrm{~cm}$, while for fayalite $l_{v}$ is of order $5 \mathrm{~cm}$ (assuming a spin period of 5 hours - see below). The thermal inertia of the body $\Gamma=(K \rho C)^{1 / 2}$ additionally enters into the calculation via a non-dimensional thermal parameter used to evaluate the weighting functions $W$ (again, see [36] for details). As can be seen from equation (5), the seasonal Yarkovsky effect is always negative, tending to decrease the orbital semi-major axis, while the diurnal component can be either negative or positive according to the value of the obliquity term $\gamma$. The thermal conductivity term $K$ is an important quantity in determining which of the diurnal or seasonal terms in equation (5) dominates. Bottke et al., [35] find that the seasonal Yarkovsky effect dominates in the high thermal conductivity domain, appropriate to an ironrich composition $(K \sim 80 \mathrm{~W} / \mathrm{m} / \mathrm{K})$, with the diurnal term becoming increasingly important in the low thermal conductivity situation appropriate to that of a body with a fayalite composition $(K \sim 4 \mathrm{~W} / \mathrm{m} / \mathrm{K})$ and/or a body having an extensive surface regolith $\left(K \sim 10^{-3} \mathrm{~W} / \mathrm{m} / \mathrm{K}\right)$. Additionally, it is also worth noting that because of the sine and cosine dependency of the obliquity term, the maximum of one term on the RHS of equation (5) corresponds to the minimum of the other.

Table 1 provides a representative set of values for the rate of change in the orbital semi-major axis due to PoyntingRoberston drag and the Yarkovsky effect. In this simulation the diameter is taken to be $D(\mathrm{~km})=1$, the spin period is set to 5 hours with the angle of obliquity being $\gamma=45^{\circ}$, and the initial orbit is set to the mid-point of the Vulcanoid zone at $d$ $(\mathrm{AU})=0.13$. The orbital decay rate due to PoyntingRobertson drag is of order $4 \times 10^{-6} \mathrm{AU} / \mathrm{Myr}$, with the compositional dependency shown in column 2 of table 1 being entirely due to the different densities of the materials being considered - as indicated by (3). For iron and fayalite compositions the orbital decay due to the seasonal Yarkovsky effect is about an order of magnitude larger than that due to Poynting-Robertson drag. In the very low conductivity $(K=$ $\left.10^{-3} \mathrm{~W} / \mathrm{m} / \mathrm{K}\right)$, low density $\left(\rho=2200 \mathrm{~kg} / \mathrm{m}^{3}\right)$ regolith situation (row 4), the orbital decay rate due to the seasonal Yarkovsky drift is comparable to that of the Poynting-Robertson drag. The diurnal Yarkovsky drift is found to vary by a factor some 300 times smaller than that of the seasonal Yarkovsky drift in the case of an iron composition, to a factor of only 5 times smaller in the case of an extensive surface regolith simulation. This latter variation is driven primarily by the change in thermal conductivity, which between the regolith and iron composition simulation increases by nearly 4 orders of magnitude.

Table 1. Characteristic absolute values for the rate of change in the semimajor axis due to Poynting-Robertson drag (PRd), the seasonal Yarkovsky effect (SYa) and the diurnal Yarkovsky effect (DYa) - the numbers in columns 2 to 4 are in units of AU/Myr. Column 1 indicates the assumed composition, and the calculation adopts common values for other terms: $P(\mathrm{hr})=5, \gamma=$ $45^{\circ}, D(\mathrm{~km})=1, d(A U)=0.13$. Column 5 indicates the thermal conductivity assumed in the various calculations.

\begin{tabular}{lllll}
\hline Composition & PRd & SYa & DYa & $\boldsymbol{K}(\mathbf{W} / \mathbf{m} / \mathbf{K})$ \\
\hline Iron & $6.5 \times 10^{-6}$ & $4.7 \times 10^{-5}$ & $1.6 \times 10^{-7}$ & 80 \\
Fayalite & $1.8 \times 10^{-6}$ & $5.8 \times 10^{-5}$ & $3.3 \times 10^{-6}$ & 4 \\
Regolith & $2.3 \times 10^{-6}$ & $2.0 \times 10^{-6}$ & $4.2 \times 10^{-7}$ & $1.0 \times 10^{-3}$ \\
\hline
\end{tabular}

Two important problems arise at this stage, and they are concerned with how to parameterize the time variation of the obliquity term, which can, in principle, vary at random between 0 and 360 degrees, and the spin-rate of the body, which again, up to a point, can vary from a large to a small value, at random, depending upon an individual object's collisional history. Bottke et al [35] assume a characteristic re-orientation timescale of order $\tau(\mathrm{yr})=1.5 \times 10^{7} R^{1 / 2}$, where the radius $R$ is expressed in meters, and presume that the spin-rate varies on the same timescale. In terms of modeling the spin period $P$, Farinella, Vokrouhlicky and Hartmann [37] suggest two possible scenarios - one has the spin-period remain fixed at $P=5$ hours, independent of body size, while the other assumes a size dependency with $P(\mathrm{hr})=5 D$, where $D$ is the diameter expressed in kilometres. The fixed 5hour spin period is essentially placed according to the rubblepile spin-up barrier which emerges from the rotation versus size data for main-belt asteroids larger than about 500-metres across. Other rotation rates, re-orientation rules and timescale could equally well apply to any given Vulcanoid. Beech and Brown [38], for example, find a size-dependent rotation rate of $P(\mathrm{hr})=2 D$ from multi-station observations of bright fireball trails - this latter rule, however, corresponds to objects with characteristic sizes smaller than a few metres across.

Even between collisions the spin period of a Vulcanoid might still increase with age as a consequence of sublimation mass loss: this effect being more efficient the smaller the heliocentric distance. The critical rotational period $P_{\text {crit }}$ at which a body is disrupted can be estimated according to equality of the centripetal and gravitational accelerations acting upon the equator of a spherical body. Accordingly: $P_{\text {crit }}=(3 \pi / G \rho)^{1 / 2}$, where $G$ is the gravitational constant and 
$\rho$ is the density. Strength-less bodies rotating with periods $P$ $<P_{\text {crit }}$ are liable to undergo rotational bursting. Indeed, rotational bursting, in principle, offers a potentially observable end phase for a Vulcanoid asteroid since the associated debris cloud will be spread over a large (sunlight reflecting) volume of space [see, e.g., 24]. For an iron composition body, $P_{\text {crit }} \sim 1.2$ hours; for a fayalite composition the critical period is $\sim 1.6$ hours. The spin-up timescale [39],

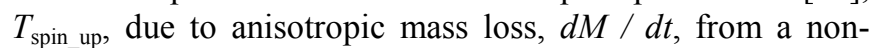
symmetric body, can be expressed as:

$$
T_{\text {spin_up }}=\frac{P}{d P / d t}=\frac{I \Omega}{N}
$$

where $I$ is the moment of inertia, $\Omega=2 \pi / P$ is the angular velocity, and $N$ is the mass-loss induced torque. Taking the object being spun-up to be a sphere with $I=2 M R^{2} / 5$ equation (8) becomes

$$
T_{\text {spin_up }}=\left(\frac{8 \pi}{9}\right) \frac{\Omega \rho R^{4}}{V K_{T}(d M / d t)}
$$

Where $K_{\mathrm{T}}$ is the dimensionless moment arm for the torque ( $K_{\mathrm{T}}=0$ for radial mass loss, and $K_{\mathrm{T}}=1$ for extreme tangential mass loss), and $V$ is the sublimation outflow speed. Jewitt [40] suggests a typical value for $K_{\mathrm{T}}$ is of order 0.05 , and we take $V$ to have a characteristic value of $200 \mathrm{~m} / \mathrm{s}$. Taking an initial spin-period of 5 hours, we find characteristically that $T_{\text {spin-up }}(\mathrm{yr}) \sim 10^{4} /(d M / d t)$, where the mass loss rate is expressed in kilograms per second. At a heliocentric distance of $0.06 \mathrm{AU}$, the inner edge of the Vulcanoid stability zone, we find through the numerical integration of equations (1) and (2), that $(d M / d t) \sim 10^{-3} \mathrm{~kg} / \mathrm{s}$ for an iron composition - for a fayalite body we find $(d M / d t)$ $\sim 10^{-5} \mathrm{~kg} / \mathrm{s}$. Accordingly, the spin-up timescale close to the inner edge of the Vulcanoid zone is of order $10^{7}$ years for an iron composition and of order $10^{9}$ years for a fayalite composition. The spin-up timescale, therefore, is of order the survival lifetime against sublimation and Poynting-Robertson drag (recall figure 3), and it would appear that an end phase involving catastrophic spin-induced disintegration is distinctly possible for those Vulcanoids that have migrated to the inner edge of the stability zone. Granvik et al. [41] have recently suggested that spin-up induced fragmentation might afford an explanation of the fewer than model-expected nearEarth asteroids having perihelia smaller than $\sim 0.1$ AU. The spin-up of asymmetric Vulcanoids, due to thermal photon induced torques, may additionally become important at larger heliocentric distances even when the sublimation mass loss is relatively small. This, so-called, Yarkovsky-O'KeefeRadzievskii-Paddack (YORP) effect [35, 39, 42] comes about through momentum transfer when a photon is reflected from the surface of an asymmetric body, and while the specific transfer is extremely small at any one instant, it is additive over time. The YORP effect is likely to be most important in the outer Vulcanoid stability zone, and then only upon the smaller, meter-sized objects. Rubincam [42] additionally points out that for slow rotators, the YORP effect may induce the onset of random tumbling modes - that is non-principle axis rotation. Random tumbling modes will tend to shutdown both the Yarkovsky and YORP effects as a consequence of the continual varying obliquity term, and this might have important consequences for the orbital evolution of objects initially located towards the outer edge of the Vulcanoid zone. In lieu of having no specific Vulcanoid shape model to work with, however, we have not investigated YORP effects in any detail, but this is an area for future study.

Tables 2 and 3 reveal the results from a series of simulations in which the lifetime against destruction for 1 and $5 \mathrm{~km}$ diameter Vulcanoids have been determined. These simulations include the effect of a varying solar luminosity, sublimation mass loss, Poynting-Robertson drag and the Yarkovsky effect. It is assumed that the rotation rate is constant at all phases of evolution, with $P=5 \mathrm{hr}$, and it is also assumed that the obliquity term remains constant. This latter assumption allows us to determine the maximum responses of the seasonal and diurnal Yarkovsky effects. Poynting-Robertson drag always acts to reduce the semimajor axis of the orbit, and when $\gamma=90$ degrees this will be enhanced by a maximum seasonal Yarkovsly drift. The seasonal Yarkovsky effect will be zero if $\gamma=0$ or 180 degrees, while the diurnal Yarkovsky effect will be at its maximum, acting to reduce the size of the orbit, when $\gamma=0^{\circ}$. The diurnal Yarkovsky drift will act to increase the orbital semi-major axis when $\gamma=180^{\circ}$. Column 6 in each table indicates that in this latter case, a primordial Vulcanoid could, in principle, survive against sublimation destruction for the entire length of the Sun's main sequence lifetime, irrespective of its initial orbit - indeed Vulcanoids in this case evolve to larger orbits over time - their lifetime is then determined according to the crossing, or not, of the resonance zones associated with Mercury and/or Venus [24, 25, 26]. When the obliquity is between $0^{\circ}$ and $90^{\circ}$ the end state is typically that of destruction due to sublimation mass loss compounded by orbit reduction. Additionally, as expected, smaller objects evolve to a final end state more rapidly than larger ones. Again, consistent with expectation, a $5 \mathrm{~km}$ initial diameter fayalite Vulcanoid could in principle survive to the present epoch if initially located towards the outer edge of the orbital stability zone. The same basic results hold true for a $5 \mathrm{~km}$ initial diameter iron Vulcanoid.

Table 2. Lifetimes against sublimation for Vulcanoids of initial diameter $D(\mathrm{~km})$ (column 1) and orbital radius $d(A U)$ (column 2). In this simulation a fayalite composition is assumed with $\rho=4390 \mathrm{~kg} / \mathrm{m}^{3}, K=3.85 \mathrm{~W} / \mathrm{m} / \mathrm{K}$, and $C=797 \mathrm{~J} / \mathrm{kg} / \mathrm{K}$ Columns 3, 4, 5 and 6 indicate the final orbital radius and the time in billions of years to acquire the end state (shown in brackets).

\begin{tabular}{llllll}
\hline $\boldsymbol{D}(\mathbf{k m})$ & $\boldsymbol{d}(\mathbf{A U})$ & $\boldsymbol{\gamma}=\mathbf{0}$ & $\gamma=\mathbf{4 5}$ & $\gamma=\mathbf{9 0}$ & $\gamma=\mathbf{1 8 0}$ \\
\hline 1 & 0.06 & $0.0(0.27)$ & $0.0(0.13)$ & $0.0(0.10)$ & $0.15(10.0)$ \\
1 & 0.13 & $0.0(4.90)$ & $0.0(1.13)$ & $0.0(0.70)$ & $0.17(10.0)$ \\
1 & 0.20 & $0.14(10.0)$ & $0.0(2.17)$ & $0.0(1.25)$ & $0.21(10.0)$ \\
5 & 0.06 & $0.0(1.17)$ & $0.0(0.63)$ & $0.0(0.47)$ & $0.10(10.0)$ \\
5 & 0.13 & $0.1(10.0)$ & $0.0(4.79)$ & $0.0(3.09)$ & $0.14(10.0)$ \\
5 & 0.20 & $0.19(10.0)$ & $0.0(8.50)$ & $0.0(5.45)$ & $0.20(10.0)$ \\
\hline
\end{tabular}


Table 3. Lifetimes against sublimation for Vulcanoids of initial diameter $D(\mathrm{~km})$ (column 1) and orbital radius $d(A U)$ (column 2). In this simulation an iron composition is assumed with $\rho=7870 \mathrm{~kg} / \mathrm{m}^{3}, K=80 \mathrm{~W} / \mathrm{m} / \mathrm{K}$, and $C=$ $500 \mathrm{~J} / \mathrm{kg} / \mathrm{K}$ Columns 3, 4, 5 and 6 indicate the final orbital radius and the time in billions of years to acquire the end state (shown in brackets).

\begin{tabular}{llllll}
\hline $\boldsymbol{D}(\mathbf{k m})$ & $\boldsymbol{d}(\mathbf{A U})$ & $\boldsymbol{\gamma}=\mathbf{0}$ & $\boldsymbol{\gamma}=\mathbf{4 5}$ & $\boldsymbol{\gamma}=\mathbf{9 0}$ & $\boldsymbol{\gamma}=\mathbf{1 8 0}$ \\
\hline 1 & 0.06 & $0.0(0.14)$ & $0.0(0.06)$ & $0.0(0.04)$ & $0.142(10.0)$ \\
1 & 0.13 & $0.0(8.19)$ & $0.0(1.02)$ & $0.0(0.57)$ & $0.134(10.0)$ \\
1 & 0.20 & $0.182(10.0)$ & $0.0(2.16)$ & $0.0(1.12)$ & $0.195(10.0)$ \\
5 & 0.06 & $0.0(0.64)$ & $0.0(0.31)$ & $0.0(0.22)$ & $0.136(10.0)$ \\
5 & 0.13 & $0.121(10.0)$ & $0.0(4.23)$ & $0.0(2.54)$ & $0.131(10.0)$ \\
5 & 0.20 & $0.197(10.0)$ & $0.0(8.02)$ & $0.0(4.97)$ & $0.199(10.0)$ \\
\hline
\end{tabular}

\section{Induction Heating}

Prior to the proto-Sun settling into its steady, hydrogenburning, main-sequence configuration it will pass through a short-lived T Tauri phase characterized by the generation of a strong, highly magnetized wind. The onset of the T Tauri phase signals the beginning of the end for the solar nebula, with the primordial dust and gas being cleared from the nebula on a timescale of order several millions of years [43]. During this clearing phase a unipolar dynamo mechanism may have operated on primordial Vulcanoids, and this in turn might have resulted in the production of significant internal heating effects $[44,45,46]$. The proto-Sun, T Tauri wind is here taken to be a fully ionized plasma, with a characteristic velocity $\mathbf{v}$, and a magnetic field $\mathrm{B}$ configured so that the electrical field $\mathrm{E}=0$ within the wind's rest frame. In the frame of any Vulcanoid asteroid, however, a motional electric field $\mathrm{E}_{\mathrm{m}}=-(\mathrm{v} / \mathrm{c}) \times \mathrm{B}$ will appear, and this may potentially drive internal heating through ohmic dissipation. An upper limit to the amount of heating is given by $\Gamma=\sigma \mathrm{E}_{\mathrm{m}}{ }^{2}$, where $\sigma$ is the conductivity of the asteroid material. Menzel and Roberge [46] indicate that under such ideal conditions the internal heating in watts per cubic meter will vary as

$$
\Gamma=\mathrm{v}^{2} B_{0}^{2} \exp (-1161 / T)
$$

where $T$ is the temperature at a distance $D$ away from the Sun, the ambient magnetic field strength $B_{0}$ is expressed in Gauss and the velocity $v$ is in units of kilometres per second. The temperature enters into equation (10) under the assumption that the material conductivity varies according to an Arrhenius-like law with temperature [46]. In a minimum mass solar nebula the temperature $T$ at distance $D(\mathrm{AU})$ from the proto-Sun will be of order that indicated by equation (1). Since we are primarily interested in the conditions that apply at the inner most edge of the solar nebula during the Sun's T Tauri phase, we take the ambient field to be that expected for a dipole configuration. Accordingly $B_{0}(D)=2000(4.654 \mathrm{x}$ $\left.10^{-3} / D\right)^{3}$, where we assume the magnetic field at the Sun's surface during the T Tauri phase is, as observations suggest, of order $2 \mathrm{kG}$, and where the distance $D$ is expressed in AU. With this formalism the ambient field in the Vulcanoid zone at $D=0.15 \mathrm{AU}$ is of order $B_{0}=0.2$ Gauss. Further into the nebular disk the magnetic field configuration will vary in a complex manner according to the ionization state of the nebula gas and the characteristic size and number density of nebula dust grains: Menzel and Roberge [46] argue, however, for a near constant field strength, of $B_{0} \sim 0.3$ Gauss, out to a distance of several astronomical units within the young solar nebula. Figure 5 shows the evaluation of equation (10) under conditions that are expected to apply in the inner solar system during the Sun's T Tauri phase. From figure 5, we see that within the Vulcanoid zone the (idealized) induction heating could amount to as much as $10^{-4}$ to $10^{-2} \mathrm{~W} / \mathrm{m}^{3}$ for typical wind velocity values. At the end of the Sun's T Tauri phase its surface magnetic field dropped by at least a factor of $10^{3}$ and from that time forward, and throughout the subsequent age of the solar system, the unipolar heating mechanism has been effectively quenched as an asteroid heating mechanism.

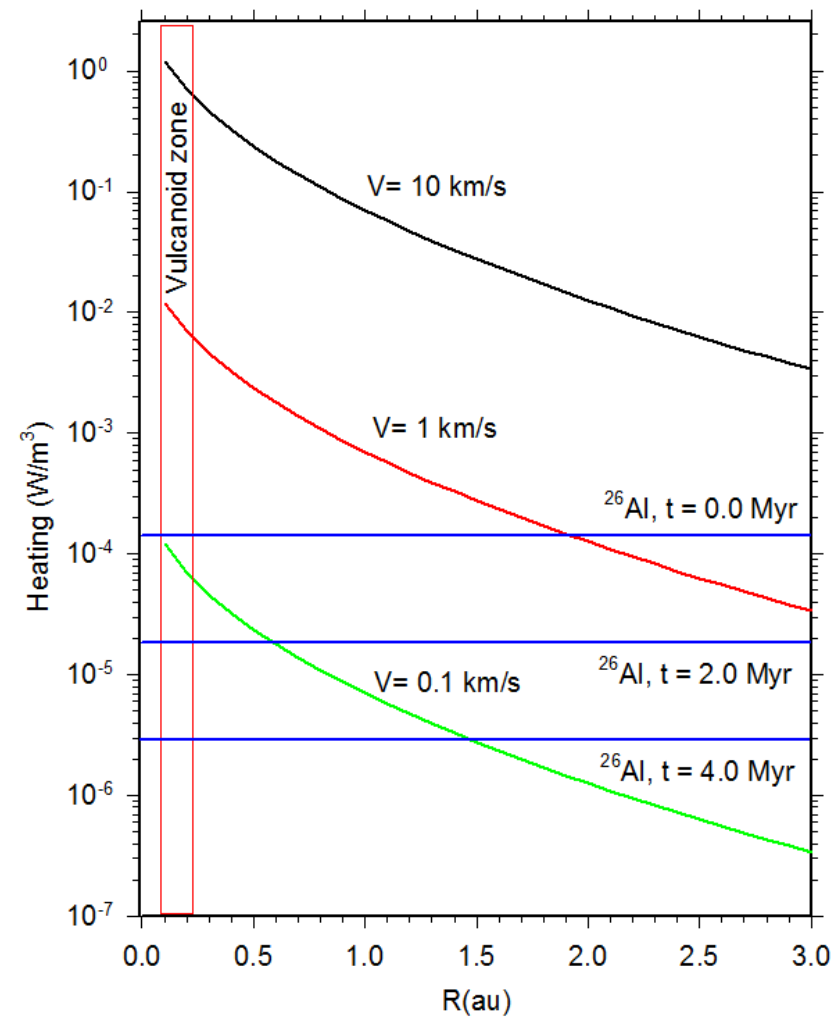

Figure 5. Induction heating of asteroids (in $\mathrm{W} / \mathrm{m}^{3}$ ), within the inner solar system, during the Sun's T Tauri phase. The typical velocity is expected to be between 0.1 and $1 \mathrm{~km} / \mathrm{s}$ with short-lived outburst speeds approaching 10 $\mathrm{km} / \mathrm{s}$. The ambient magnetic field strength is taken to be $B_{0}=0.2$ Gauss. The Vulcanoid zone is highlighted on the left-hand side of the diagram, and the three horizontal (blue) lines indicate the expected internal heating due to the decay of ${ }^{26} \mathrm{Al}$ at the time of planetesimal formation and at approximately 2 and 4 half-lifetimes later.

The amount of energy deposited into the asteroid's interior over the time interval $\Delta \mathrm{t}$ will be of order $\Delta \mathrm{Q}=\Gamma \Delta \mathrm{t}$, and given a heat capacity $C$ and material density $\rho$, the temperature change in the interior $T_{\text {int }}$ of the asteroid will be of order $\Delta \mathrm{Q}=\rho C\left(T_{\mathrm{int}}-T\right)$. Taking a specific heat capacity of $600 \mathrm{~J} / \mathrm{kg} / \mathrm{K}$ and a density of $4390 \mathrm{~kg} / \mathrm{m}^{3}$ (values typical of fayalite), we find that the interior of a primordial Vulcanoid asteroid could be driven close to its melting point $\left(T_{\text {melt }} \sim\right.$ $1800 \mathrm{~K}$ for fayalite) within $10^{4}$ years when $v=1 \mathrm{~km} / \mathrm{s}$, and within $10^{6}$ years when $v=0.1 \mathrm{~km} / \mathrm{s}$. These values, however, 
recall, are based upon the assumption of ideal circumstances and maximal induction heating. Remarkably, the idealized induction heating effect in the Vulcanoid zone (in $\mathrm{W} / \mathrm{m}^{3}$ ) dominates that expected from the decay of ${ }^{26} \mathrm{Al}$ once the velocity of the Sun's T Tauri wind exceeds a few hundreds of meters per second. These calculations suggest that any primordial Vulcanoids may well develop differentiated interiors soon after formation, and this, of course, will have a significant effect upon their future evolution. Indeed, by the time of the Late Heavy Bombardment (at a solar system age $\sim 800$ million years) it might well be expected that the majority of primordial Vulcanoids will have developed an internal structure consisting of a nickel-iron core surrounded by a silicate-rich, basaltic mantle.

\section{Collisional History}

As with any population of objects having similar initial orbits subject to varying degrees of dynamical evolution, Vulcanoid upon Vulcanoid collisions are expected to occur [29]. Likewise, collisions between Vulcanoids and asteroids and cometary nuclei, the latter having evolved very small perihelia, are also possible [24, 41]. At earlier epochs an initial Vulcanoid population would have been subject to collisions with material associated with the Late Heavy Bombardment, and possible from material produced by inner-planet disruption if the TPP [22] scenario played out within our solar system.

The characteristic encounter velocity for a swarm of objects having random orbits is given by Stern and Duda [29] as

$$
V_{\text {enc }}(\mathrm{km} / \mathrm{s})=180<e>\sqrt{\frac{0.1(A U)}{a}}
$$

where $\langle e\rangle$ is the average orbital eccentricity and $a$ is the orbital semi-major axis of the target object. For objects in the Vulcanoid zone, where $0.06<a(\mathrm{AU})<0.2$, it can be seen from equation (11) that the encounter velocity is going to be very high for all but the smallest of eccentricity encounters. When $e=0.01$, for example, the encounter velocity will be of order $2 \mathrm{~km} / \mathrm{s}$ in the inner most region of the Vulcanoid zone and about $1 \mathrm{~km} / \mathrm{s}$ at the outer edge. These encounter velocities are several orders of magnitude larger than a typical escape velocity $V_{\mathrm{esc}}=(2 G M / R)^{1 / 2}$. Indeed, for a 10$\mathrm{km}$ diameter Vulcanoid, $V_{\text {esc }} \sim 10 \mathrm{~m} / \mathrm{s}$, and it is expected, therefore, that collisions will be highly erosive with little to no impact ejected material falling back to the target body surface. At face-value this result would argue against Vulcanoids developing extensive regoliths at their surface. Such may be the case, but we note that other regolith development mechanisms may be at play within the Vulcanoid zone. Specifically, fragmentation due to thermal fatigue is likely to be an efficient mechanism for the break-up of Vulcanoid surface material. Delbo et al., [47] have recently argued that at $1 \mathrm{AU}$ from the Sun the survival time against thermal cracking, for a $10-\mathrm{cm}$ sized boulder, is as short as 1000 years. It is presently not clear how such arguments might relate to the Vulcanoid asteroids, which will certainly undergo more intense temperature cycling than that of a main-belt asteroid. If Vulcanoids are able to develop deep surface regoliths via thermal fatigue and cracking then the consequences for their orbital evolution could be profound with respect to the reduced efficiency of the Yarkovsky drift mechanism (as described in section 3 and illustrated in table 1). Likewise it is now recognized that objects supporting deep regolith regions and possessing a high degree of internal porosity can survive disruption against impacts to much higher energies than those having a monolithic constitution. This latter situation has recently been reviewed in detail by Syal et al. [48] who investigate the survival of the $22 \mathrm{~km}$ diameter moon Phobos against the formation of its largest impact crater - the $9 \mathrm{~km}$ diameter Stickney. Essentially, they find, it is the relatively high porosity of the target body that helps keep it intact during what would otherwise be a highly disruptive impact. Internal porosity can only help maintain system integrity up to some critical level, however, since at some point gravity and cohesive internal forces will no longer act to stave-of catastrophic break-up. Indeed, Granvik et al. [49] have argued that the dearth of low-albedo near-Earth asteroids having very small perihelion distances is a result of such objects undergoing catastrophic break-up once their perihelion distance falls below 0.06 AU. The Sun's Roche limit for an object held together purely by the mutual gravitational attraction of is sub-components is of order $R_{\text {Roche }} \approx 0.01 \mathrm{AU}$, and this suggests that the tidal disruption of highly fragmented objects may be an important lossmechanism at the inner-most boundary of the Vulcanoid zone. The possibility of catastrophic crumbling is further enhanced if one additionally allows for the relatively rapid, that is of-order one billion-years, rotation spin-up timescale [recall equation (9)], for those objects located close to inneredge of the Vulcanoid zone.

A measure of the typical collision time $t_{\text {col }}$ in the Vulcanoid zone can be expressed in terms of a collision cross-section area $\sigma=\pi R^{2}$, where $R$ is the radius, the encounter velocity $V_{\text {enc }}$ and the number density of Vulcanoids per unit area $N_{\mathrm{V}}$, with $t_{\text {col }}=1 / \sigma V N_{\mathrm{V}}$. With reference to figure 2 of [24] the volume of the Vulcanoid zone is of order $0.015 \mathrm{AU}^{3}$, and following Stern and Durda [29] by postulating an original population of say $10^{4}$ objects, so $N_{\mathrm{V}} \sim 2 \times 10^{-28}$ objects per cubic meter. Adopting a typical collision velocity of $15 \mathrm{~km} / \mathrm{s}$, corresponding to $\langle e\rangle=0.1$, so the collision time will be of order $540 \mathrm{Myr}$ for a $5 \mathrm{~km}$ diameter Vulcanoid, and of order $135 \mathrm{Myr}$ for a $10 \mathrm{~km}$ diameter Vulcanoid. The collision time for objects having a diameter of order $1 \mathrm{~km}$ is comparable to the present age of the solar system (4.5 billion years). Purely dependent (at this stage) upon the assumed initial population of objects, $N_{\mathrm{V}}$, the characteristic erosion lifetime of multikilometre sized Vulcanoids is expected to vary from several $10^{7}$ to several $10^{9}$ years.

In order to estimate the population evolution Stern and Durda [29] used a multi-zone collision rate model that 
computes orbit-averaged collision rates combined with a disruption model dependent upon assumed parameters for material strength and fragment production. While a whole series of simulations were run under variously assumed scenarios, Stern and Durda [29] found, as expected, that the largest objects were soon eroded by collisions, but in all of their simulations a few objects with sizes of order several kilometres did manage to survive to the present age of the solar system. Collisions in the inner region of the Vulcanoid zone tended to be more disruptive, because of the higher encounter velocities there, and the majority of surviving multi-kilometre-sized objects were located towards the outer edge of the Vulcanoid zone at a heliocentric distance of 0.2 AU.

Depending upon ones sense of optimism it can be conclude from the analysis presented by Stern and Durda [29] that there are either no large, that is of order a few kilometres in size, primordial Vulcanoids in the solar system at the present epoch, or that there might yet be a few lucky survivors. Certainly the implications are clear that due to the highly erosive collisions that will take place within the Vulcanoid zone, followed by the relatively rapid removal of small fragments via Poynting-Roberston drag and Yarkovsky drift, any primordial population of Vulcanoids will have been severely depleted by the present epoch. None-the-less, there is additionally no reason to fully suppose that there are absolutely no kilometre-sized survivors, and this is why continued observational survey work is going to be essential for resolving the Vulcanoid narrative.

\section{Conclusions}

In spite of some 150 years of debate and observations it is still not clear if a population of Vulcanoid asteroids exists at the present time $[1,4,12,50,51,52,53]$. The theoretical situation, as far as can be read from past publications, is such that there are no inescapable and/or overriding physical reasons to fully preclude the existence of Vulcanoid asteroids either in the past or at the present epoch. Certainly, however, the studies that have been published on the topic suggest that should a population of Vulcanoids actually exist at the present time then it will be neither extensive with respect to total number and that at best its largest members might be just a few kilometres across. Additionally, however, the available observations do preclude the existence of any Vulcanoids larger than about $10-\mathrm{km}$ in diameter at the present epoch. There are many naturally occurring mechanisms that will tend to deplete a primordial Vulcanoid population collisions are certainly going to be important, but so too are the various radiative, induction heating and spin-up effects that have been described. Poynting-Robertson drag, due to the high solar irradiance experienced, will be very effective at removing small material from the inner Vulcanoid region. The Yarkovsky effect will additionally aid in this removal, or, it may potentially act to preserve a Vulcanoid's orbit according to the specific and individual spin rates and obliquities that chance to apply. At the present time, however, there are no good models, nor good observational data to guide the researcher in making any clear-cut statement about the spin-evolution of Vulcanoid asteroids, and nor are there any specific data to help constrain questions relating to material strength, surface regolith formation, and indeed, chemical composition and internal structure.

It remains a fact that the Vulcanoids are a hypothetical group of objects still wanting a single positive observational candidate, and it is also true, from the survey data that presently exists, that the room for theoretical maneuvering is becoming increasingly small. The inherent observational problem with the Vulcanoid region is its close proximity to the Sun - indeed, the Vulcanoids are located in the very zone that astronomers, outside of the short time interval associated with total solar eclipse events, have historically avoided looking at. This may change in the future, although there is no good justification (one's personal preferences aside) for launching a dedicated spacecraft mission to look for Vulcanoids. Surveys such as that projected for the Sentinel mission [28] and perhaps the Solar Orbiter stand the best chance for the space-based detection of Vulcanoids in the near future, although neither mission is specifically targeting the Vulcanoid zone for study. Serendipity may yet favour us with a find, but the time is also rapidly approaching when the deduced upper size limit to any potential Vulcanoid will be pushed to a level below which it will be reasonable to conclude that there are no such objects at the present epoch. In his 1953 Astronomical Society of the Pacific Leaflet on the planet Vulcan narrative [51], American astronomer Olin J. Eggen noted that, "the mathematical necessity for Vulcan was established by a French astronomer [Le Verrier] at his desk in Paris [in 1859]. The necessity was removed by a German mathematician [Einstein] at his desk in Berlin, 500 miles distant and 56 years later [in 1915]". We have now passed the first centennial of Einstein's introduction of general relativity, and while there is now no "necessity" for any substantive object to be orbiting interior to Mercury, the question concerning the existence, or not, of Vulcanoid asteroids continues unanswered (both at the desk and in the observatory) and will only be resolved through continued survey work (see e.g., [53, 54]).

One of the key physical insights developed during the course of this new study concerns the importance of including the effects of the Sun's varying luminosity on Vulcanoid evolution. Indeed, over the lifetime of the solar system the Sun's luminosity has increased by some $30 \%$, and this will have had a dramatic effect upon potential Vulcanoid survivability. The Sun's luminosity, as we have seen, is fundamental to the determination of the location of the inneredge of the Vulcanoid zone, via sublimation effects, and it is also fundamental in determining the magnitude of the Poynting-Robertson drag and Yarkovsky drift. The smaller solar luminosity in the younger solar system does not change the collisional history of the Vulcanoid zone, but it significantly changes the thermal and radiative clearing scenarios. Additionally, we have found that induction heating, during the Sun's T Tauri phase, may have been 
highly important in heating the interiors of any primordial Vulcanoids, resulting in their internal differentiation. Studies relating to the evolution of hypothetical Vulcanoid populations, including the effects of collisions, sublimation, radiation interactions and solar evolution are presently being pursued and will be presented at a later date.

\section{References}

[1] Le Verrier, U. J. J. 1859. Lettre de M. Le Verrier á M. Faye sur la tháorie de Mercure et sur le movement du párihália de cette planáte. Comptes rendus hebdomadaires de séances de l'Académie des sciences. Vol. 49, 379-383.

[2] Hall, Asaph. 1894. A suggestion in the theory of Mercury. Astron. J. 14, 49-51.

[3] Beech, M. 2014. The Pendulum Paradigm - Variations on a Theme and the Measure of Heaven and Earth. Brown Walker Press, Boca Raton, Florida. pp. 44-50.

[4] Baum, R., and Sheehan, W. 1997. In Search of Planet Vulcan - the ghost in Newton's clockwork universe. Plenum Press, New York.

[5] Pannekoek, A. 1961. A History of Astronomy. Interscience Publishers, inc. New York. pp. 359-363.

[6] Nieto, M. 1972. The Titius-Bode law of planetary distances: its history and theory. Pergamon Press, Oxford.

[7] Jaki, S. L. 1972. The Original Formulation of the Titius-Bode Law. J. Hist. Astron. 3, 136-138.

[8] Jenkine, B. G. 1878. Vulcan and Bode's Law. Nature, 19, 7475 .

[9] P. E. Chase, 1873. Note on Planeto-Taxis. Publications of the American Philosophical Society, 13, 143 - 145.

[10] Hayes, W., and Tremaine, S. 1998. Fitting selected random planetary systems to Titius-Bode laws. Icarus, 135, 549-557.

[11] Schumacher, G., and Gay, J. 2001. An attempt to detect Vulcanoids with SOHO/LASCO images I: Scale relativity and quantization of the solar system. $A \& A$. 368, 1108-1114.

[12] Levenson, T. 2015. The Hunt for Vulcan. Random House, New York.

[13] Challis, J. 1860. On the planet within the Orbit of Mercury, discovered by M. Lescarbault. Cambridge Phil. Soc. Proc. 1, 219-222.

[14] Russell, H. N., Dugan, R. S., and Stewart, J. Q. 1926. Astronomy 1: Solar System. Ginn \& Co., Boston. p. 358.

[15] Young, C. 1899. A Text-Book of General Astronomy for Colleges and Scientific Schools. Ginn \& Co. Boston. pp. 373375 .

[16] Courten, H. C., Brown, D. W., and Albert, D. B. 1976. Ten Years of Solar eclipse comet Searches. BAAS. 8, 504.

[17] Leake, M. A., Chapman, C. R., Weidenschilling, S. J., Davis, D. R., and Greenberg, R. 1987. The chronology of Mercury's geological and geophysical evolution - the Vulcanoid hypothesis. Icarus, 71, 350-375.
[18] Durda, D. D., et al., 2000. A New Observational Search for Vulcanoids in SOHO/LASCO Coronagraph Images. Icarus, $148,312-315$.

[19] Zhao, H., Lu, H., Zhaori, G., Yao, J., and Ma, Y. 2009. The search for vulcanoids in the 2008 total solar eclipse. Sci. China Ser G. 52, 1790-1793.

[20] Steffl, A. J., et al. 2013. A search for Vulcanoids with the STEREO Heliospheric Imager. Icarus, 223, 48-56.

[21] Strom, R. G. 2015. The inner solar system cratering record and the evolution of impactor populations. Res. Astron. Astrophys. 15, article id 407.

[22] Volk, K., and Gladman, B. 2015. Consolidating and crushing exoplanets: did it happen here? Astrophs. J. Lett. 806, article id L26.

[23] Warell, J., Karlsson, O., and Skoglov, E. 2003. Evolution of Mercury-like orbits: a numerical study. $A \& A$. 411, 291-307.

[24] Beech, M., and Peltier, L. 2015. Vulcanoid asteroids and sungrazing comets - past encounters and possible outcomes. Am. J. Astron. Astrophys. 3 (2), 26-36.

[25] Evans, N. W., and Tabachnik, S. A. 1999. Possible long-lived asteroid belts in the inner Solar System. Nature, 399, 41-43.

[26] Evans, N. W., and Tabachnik, S. A. 2002. Structure of Possible Long-lived Asteroid Belts. MNRAS. 333, L1-L5.

[27] Wieczorek, M. A., et al. 2011. Mercury's spin-orbit resonance explained by initial retrograde and subsequent synchronous rotation. Nature Geoscience, 5, 18-21.

[28] Buie, M. W., Reitsema., and Linfield, R. P. 2016. Surveying the Inner Solar System with an Infrared Space Telescope. Arxiv: 1607.05255.

[29] Stern, S. A., and Durda, D. D. 2000. Collisional evolution in the Vulcanoid region: implications for present-day population constraints. Icarus, 143, 360-370.

[30] Vokrouhlicky, D., Farinella, P., and Bottke, W. F. 2000. The depletion of the putative Vulcanoid population via the Yarkovsky effect. Icarus, 148, 147-152.

[31] Beech, M., and Peltier, L. 2015. Lifetime against sublimation and an initial mass estimate for the exoplanet $\alpha \mathrm{Cen} \mathrm{Bb.} \mathrm{Am}$. J. Astron. Astrophys. 3 (4), 70-76.

[32] Townsend, R. 2015. http://www.astro.wisc.edu/ townsend/static.php?ref=ez-web.

[33] Wyatt, S. P., and Whipple, F. L. 1950. The PoyntingRobertson effect on meteor streams. Astron. J. 111, 134-141.

[34] Burns, J. A., Lamy, P. I., and Soter, S. 1979. Radiation forces on small particles in the solar system. Icarus, 40, 1-48.

[35] Bottke, W., Vokrouhlicky, D., Rubicam, D. P., and Nesvorny, D. 2006. The Yarkovsky and YORP effects: implications for asteroid Dynamics. Ann. Rev. Earth Planet Sci., 34, 157-191.

[36] Vokrouhlicky, D. 1998. Diurnal Yarkovsky effect as a source of mobility of meter-sized asteroidal fragments. MNRAS. 335, 1093-1100.

[37] Farinella, P., Vokrouhlicky, D., and Hartmann. 1998. Meteorite delivery via Yarkovsky orbital drift. Icarus, 132, 378-387. 
[38] Beech, M., and Brown, P. 2000. Fireball flickering - the case for indirect measurement of meteoroid rotation rates. Plan. Space Sci. 48, 925-932.

[39] Samarasinha, N. H. 2008. Rotational excitation and damping as probes of interior structures of asteroids and comets. Meteoritics and Plan. Sci. 43, 1063-1073.

[40] Jewitt. D. C. 2004. From cradle to grave: the rise and demise of comets. In Comets II (Eds. M. C. Festou; H Uwe Keller; H. A. Weaver). University of Arizona Press, Tucson. pp. 659-676.

[41] Granvik, M., et al., 2016. Super-catastrophic disruption of asteroids at small perihelion distances. Nature, 530, 303-306.

[42] Rubincam, D. P. 2000. Radiative spin-up and spin-down of small asteroids. Icarus, 148, 2-11.

[43] Wang, H., el al., 2017. Lifetime of the solar nebula constrained by meteorite paleomagnetism. Science, 355, 623-627.

[44] Sonnett, C. P., Colburn, D. S., and Schwarte, K. 1968. Electrical heating of massive parent bodies and planets by dynamo induction from pre-main sequence $\mathrm{T}$ Tauri solar wind. Nature, 219, 924-926.

[45] Herbert, F. 1989. Primordial electrical induction heating of asteroids. Icarus, 78, 402-410.

[46] Menzel, R., and Roberge, W. G. 2013. Reexamination of induction heating of primitive bodies in protoplanetary disks. Ap. J. 776, article id. 89.
[47] Delbo, M., et al. 2014. Thermal fatigue as the origin of regolith on small asteroids. Nature, 508, 233-236.

[48] Syal, M. B., Rovny, J., Owen, J. M., \& Miller, P. L. 2017. Excavating Stickney Crater at Phobos. 2016. Geophysical Research Letters, 43, 10,595-10,601.

[49] Granvik M., et al. 2016. Super-catastrophic disruption of asteroids at small perihelion distances. Nature, 530, $303-$ 306.

[50] Swift, L. 1878. Discovery of Vulcan. Nature, 18, 539.

[51] Eggen, O. J. 1953. Vulcan. Astron. Soc. Pacific Leaflets, 6, No. 287.

[52] Frontenrose, R. 1973. In search of Vulcan. Jour. Hist. Astron. $4,145-158$

[53] Merline, W. J. et al. 2016. Search for Vulcanoids and Mercury satellites from MESSENGER. 47 $7^{\text {th }}$ Lunar and Planetary Science Conference, 2765. pdf.

[54] Stern, S. A., Durda, D. D., Davis, M., and Olkin, C. B. 2010. Planetary Science from a Next-Gen Suborbital Platform: sleuthing the long sought after Vulcanoid asteroids. NextGeneration Suborbital Researchers Conference, Boulder. 4004. pdf. 